\title{
Une re-vue de la sénescence cellulaire
}

> n considère à l'heure actuelle que la sénescence cellulaire, tout comme l'apoptose, représente un véritable mécanisme de suppression des tumeurs chez les mammifères. La sénescence cellulaire a été identifiée à l'origine comme phénomène consécutif au raccourcissement télomérique et limitant la durée de vie des cellules humaines en culture primaire. Depuis, de nouvelles données ont établi qu'à côté de cette sénescence réplicative (RS) existait également la sénescence prématurée induite par le stress (SIPS) dont le phénotype est impossible à distinguer de celui de la RS. La SIPS peut être induite aussi bien dans des cellules normales que dans des cellules tumorales en l'absence de nombreux cycles de division cellulaire, et ce en réponse à divers stress et/ou signaux inappropriés. En dépit de l'activité oncosuppressive potentielle du processus de sénescence, des résultats récents indiquent que les cellules sénescentes pourraient, dans certaines circonstances, favoriser l'émergence de cellules tumorales ou promouvoir la croissance de cellules prénéoplasiques. La sénescence agirait alors en véritable «cheval de Troie » biologique. Nous tenterons dans cet article de faire le point des connaissances actuelles sur les causes et les conséquences de la sénescence cellulaire in vitro et in vivo. <

\section{Ami ou ennemi}

\section{de la promotion tumorale?}

Oliver Bischof, Anne Dejean, Pascal Pineau

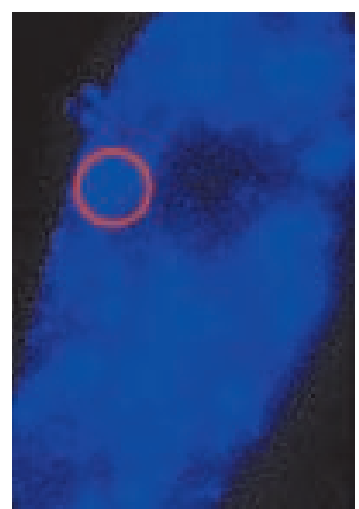

Unité d'Organisation Nucléaire et Oncogenèse (ONO), Inserm U579, Institut Pasteur, 28, rue du Docteur Roux, 75724 Paris Cedex 15, France. obischof@pasteur.fr

les perturbations chromatiniennes, le stress oxydant et le dysfonctionnement télomérique [1]. Les télomères consistent en des séquences d'ADN répétitives situées aux extrémités chromosomiques, protégées par la «shelterin», un complexe protéique qui inhibe la dégradation ou les fusions chromosomiques induites par les systèmes de réparation de l'ADN. Au cours de chaque cycle réplicatif, les télomères raccourcissent jusqu'à une limite au-delà de laquelle ils ne sont plus fonctionnels (limite de Hayflick), ce qui déclenche alors le signal de sénescence [2]. Ce processus, également appelé sénescence réplicative (RS), contraste avec l'induction de la sénescence par d'autres stress, qui ne nécessitent pas de divisions cellulaires; on désigne ce processus sous le terme de sénéscence prématurée induite par le stress (SIPS). Le fait que les différents types de stress soient potentiellement tumorigènes a fait naître l'idée que la sénescence est un mécanisme majeur d'inhibition de la prolifération dans des cellules normales exposées à un risque de transformation [3, 4]. Cette réponse cellulaire de type «sénescence» ne se limite pas aux cellules normales mais s'applique également à certaines cellules tumorales exposées aux anticancéreux, soulignant ainsi que le programme de sénescence dans les cellules cancéreuses existe à l'état dormant mais est réactivable par des signaux appropriés $[5,6]$.

Quel que soit le stimulus qui la déclenche, la sénescence signifie - et c'est ce qui la différencie de la quiescence - 
qu'il se produit un arrêt apparemment permanent du cycle cellulaire. Les cellules quiescentes (par exemple des cellules qui ont arrêté de proliférer en réponse à un sevrage en facteur de croissance ou à une inhibition de contact) reprennent leur prolifération en réponse à un signal mitogène alors que les cellules sénescentes ne peuvent être stimulées par aucun des stimulus physiologiques connus. Lors de la sénescence, l'arrêt se produit généralement au cours de la phase $\mathrm{Gl}$ du cycle cellulaire (alors que les cellules quiescentes s'arrêtent en G0). Toutefois, en fonction du stimulus déclencheur, une proportion significative des cellules peut également entrer en sénescence au cours des phases $S$ ou G2. L'arrêt du cycle cellulaire est généralement accompagné d'une augmentation de la résistance à certains signaux apoptotiques ainsi que de l'augmentation de l'expression de l'activité $\beta$-galactosidase lysosomiale (SA- $\beta$-Gal). De plus, la sénescence est caractérisée par de profondes modifications cytomorphologiques - aplatissement cellulaire, vacuolisation du cytoplasme, augmentation du volume nucléaire (x 5 ou 10) (Figure 1) - ainsi que par des altérations de l'architecture chromatinienne et de l'expression génétique [45].

\section{Modifications des régulateurs du cycle cellulaire au cours de la sénescence}

On observe dans les cellules sénescentes des changements très importants de l'expression d'activateurs et d'inhibiteurs du cycle cellulaire, de facteurs inflammatoires ou immunomodulateurs, et de certains membres de la famille des insulin-like growth factor binding protein (IGFBP) [1]. En parallèle, l'expression des inhibiteurs des kinases dépendantes des cyclines (CKI), p14/19ARF, p15, pl6 et p21, composants cruciaux des réseaux mettant en jeu $\mathrm{p} 53$ et $\mathrm{Rb}$ (protéine du rétinoblastome), est élevée dans les cellules sénescentes [7]. Or, les fonctions de p53 et $\mathrm{Rb}$, deux régulateurs transcriptionnels, sont fréquemment altérées dans les cellules cancéreuses à tel point qu'ils sont considérés comme les archétypes des protéines oncosuppressives chez les mammifères [8] (Figure 2). p53 module de façon directe et positive l'expression de p21 mais le mécanisme qui régule p16 n'est que partiellement élucidé. L'activité inhibitrice de la croissance qu'exercent ces deux protéines est principalement régulée par des modifications post-traductionnelles incluant la phosphorylation, l'acétylation, l'ubiquitinylation ainsi que la sumoylation. Les modifications post-traductionnelles massives qui affectent p53 suggèrent la possibilité d'un code de modification de p53 (et/ou Rb) similaire au code des histones. Ce code pourrait conditionner l'orientation de la cellule vers la sénescence, l'apoptose ou l'arrêt transitoire de croissance [9-11]. Bien que les activités des CKI ne soient pas toutes équivalentes, on sait que, pour l'essentiel, ces inhibiteurs maintiennent $\mathrm{Rb}$ dans un état hypophosphorylé, donc actif. L'axe p53-p21 est le médiateur principal de la sénescence induite par les télomères ou le stress génotoxique, alors que l'axe pl6-Rb est, quant à lui, responsable des différentes formes de sénescence induites par d'autres substances que les seules substances génotoxiques [12, 46] (Figure 2). La forme active de Rb, en inhibant l'action du facteur de transcription $\varepsilon 2 F$, réprime la transcription de gènes permettant la progression du cycle cellulaire comme PCNA (proliferation cell nuclear antigen) ou les cyclines $A, B$ et $\varepsilon$. L'inhibition de $\varepsilon 2 F$ dans les cellules sénescentes est associée à des changements d'architecture chromatinienne caractérisés par des ponctuations multiples de l'ADN appelé SAHF (pour foyers d'hétérochromatine associés à la sénescence). La formation de ces foyers dépend de l'état fonctionnel du gène $R b$, ainsi que de l'expression des protéines de la famille HMG (high mobility group), de PML (promyelocytic leukemia), de PIASy (protein inhibitor of activated STAT) et de certains chaperons d'histones. Les SAHF comportent plusieurs marqueurs d'hétérochromatine transcriptionnellement réprimée comme HPl (heterochromatin protein 1) ou les formes di- et tri-méthylées sur la lysine 9 de l'histone H3 (H3K9me2/3) [9-11]. Bien qu'aucune preuve solide ne soit disponible à ce jour, on pense que la formation de ces foyers bloquerait les cellules de façon irréversible dans un état sénescent en réprimant tout particulièrement les cibles de $\varepsilon 2 F$. Enfin, élément nouveau sur lequel nous reviendrons, l'on observe dans certains cas un accroissement du potentiel sécrétoire des cellules sénescentes, phénomène que l'on désigne sous le terme de phénotype sécrétoire associé à la sénescence ou SASP.

\section{Foyers de lésion de l'ADN}

Les cellules sénescentes peuvent également comporter deux autres types d'altérations: des foyers de lésion de l'ADN associés à la sénescence ou SDF (senescenceassociated DNA damage foci) $[13,14]$ et, dans les cellules en sénescence réplicative, des foyers télomériques dysfonctionnels associés à la sénescence (ou TIF, telomere dysfunction-induced foci) [15-17]. SDF et TIF sont tous deux caractérisés par leur colocalisation dans des foyers de protéines signalant les lésions de l'ADN comme $\gamma \mathrm{H} 2 \mathrm{AX}$, ATM ou 53BP1. Les SDF sont particulièrement visibles au cours de la sénescence induite par les oncogènes (comme RAS oncogénique) qui reproduit les étapes de la réponse aux lésions de l'ADN dépendante de p53 engendrée par le stress réplicatif. La fonction précise des SDF dans la sénescence est sujette à controverse car les différentes approches expérimentales n'ont pas toutes démontré que la destruction des SDF aboutissait à la reprise du cycle cellulaire. Ce phénomène pourrait en définitive être lié à la capacité qu'aurait la cellule de mobiliser ou pas l'axe pl6-Rb (voir plus loin).

\section{À la recherche de biomarqueurs spécifiques}

Le phénotype sénescent comporte donc des éléments caractéristiques (Figures 1 et 3 ). Toutefois, tous les changements énumérés ci-dessus ne sont pas présents 


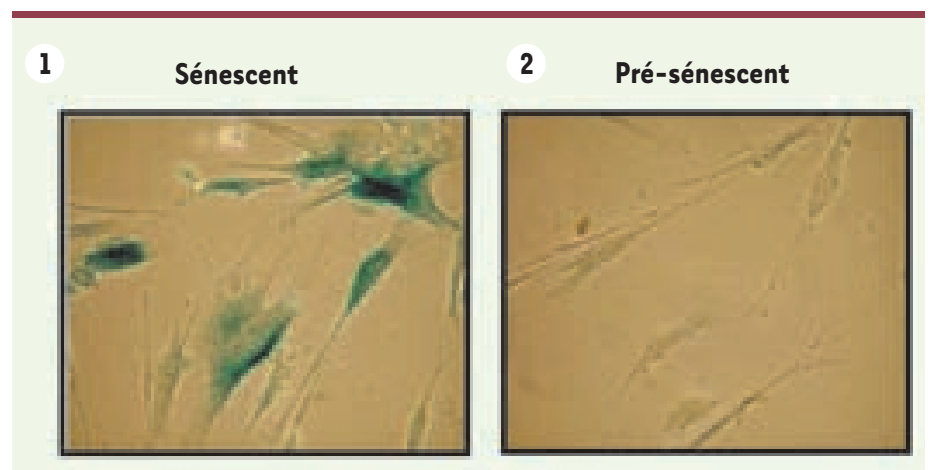

3

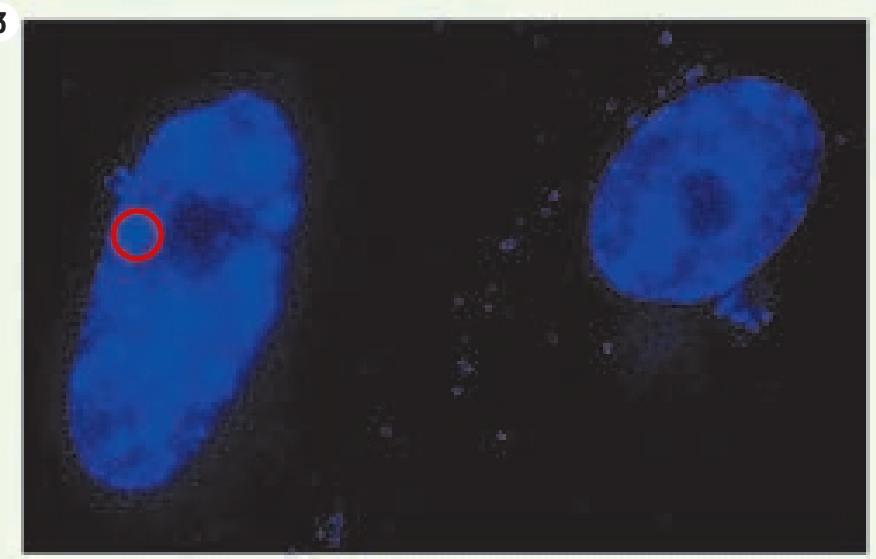

Figure 1. Caractères de la sénescence cellulaire. Comparaison des altérations cytomorphologiques et des marqueurs biochimiques observés dans un fibroblaste humain sénescent (induit par la forme oncogénique de RAS) (1) et un fibroblaste pré-sénescent (2). SA- $\beta$-Gal, $\beta$-galactosidase associée à la sénescence; SASP, phénotype sécrétoire associé à la sénescence; SAHF, foyers hétérochromatiniens associés à la sénescence; TIF, foyers lésionnels induits par les télomères dysfonctionnels; SDF, foyers de lésion de I'ADN associés à la sénescence. Le cercle rouge dans la figure du bas (3) entoure un foyer d'hétérochromatine associé à la sénescence (SAHF) tels qu'on peut les voir après une coloration au DAPI. $(1,2)$ Modifications cytomorphologiques. Cellules aplaties, cytosol vacuolisé, aspect granuleux accentué. (3) Altérations moléculaires et biochimiques. Expression génétique (ex. p15, p16, p21, PML, uPAI) : sécrétion accrue (ex. MMP, cytokines: SASP), résistance à l'apoptose (dépendante du type cellulaire), positivité pour la SA- $\beta$-galactosidase (coloration bleue). (3) Changements infranucléaires. Condensation chromatinienne: SAHF, concentration focale en $\mathrm{H} 3 \mathrm{~K} 9 \mathrm{me}_{2}$ et HPl (cercle rouge). Foyers de concentration des protéines de réparation comme ATM ou $\gamma \mathrm{H} 2 \mathrm{Ax}$ au niveau de lésions de I'ADN : SDF, au niveau des télomères: TIF. dans tous les types cellulaires étudiés. En réalité, on manque toujours de biomarqueurs spécifiques de l'état de sénescence, les marqueurs généralement utilisés pour l'identifier in vivo étant plutôt des marqueurs qui lui sont associés (de dysfonction télomérique, de lésions de l'ADN). Finalement, nous voudrions insister sur le fait qu'il existe des différences entre tissus et entre espèces quant à l'importance respective des voies p53 ou Rb pour l'induction de la sénescence [18]. Alors que dans certains fibroblastes humains, la voie p53 joue un rôle majeur, dans d'autres c'est la voie $\mathrm{Rb}$, alors que dans une troisième catégorie les deux voies fonctionnent conjointement. Chez la souris, c'est la p53 qui agit de façon prédominante pour induire la sénescence, mais là encore des exceptions existent (voir plus loin pour plus de détails).

\section{Pertinence physiologique de la sénescence dans la suppression des tumeurs}

II y a peu encore, l'idée selon laquelle la sénescence, comme l'apoptose, était un mécanisme de suppression des tumeurs pertinent in vivo manquait d'arguments car elle se fondait uniquement sur les observations faites dans des cultures cellulaires in vitro. Des preuves solides sont maintenant apparues grâce aux publications assez retentissantes de plusieurs modèles murins in vivo faisant appel aux techniques de knock-in et knock-out. Ces études ont montré que le stress oncogénique sous la forme de l'hyperactivité d'un oncogène ou de la perte d'un gène suppresseur de tumeur, produisait des lésions bénignes plutôt que malignes et que ces lésions étaient constituées de cellules sénescentes. Comme on pouvait s'y attendre, des tumeurs malignes ayant inactivé ou court-circuité le programme de sénescence se développèrent à partir des lésions bénignes [19-23]. Au cours d'une étude pionnière, Carol Greider et Sandy Chang fournirent les preuves que la sénescence réplicative se produit in vivo et réduit le développement de cancers de façon significative [24-26]. Poursuivant dans cette direction, Clemens Schmitt et ses collaborateurs démontrèrent en 2002, en utilisant un modèle murin de lymphome de Burkitt, que dans des cellules cancéreuses où la réponse apoptotique était abrogée, des agents génotoxiques pouvaient encore déclencher la sénescence [6]. Des résultats semblables ont été publiés par Robert te Poele au cours de la première étude portant sur le cancer du sein humain [27]. Cet auteur a montré que les cellules des tumeurs mammaires devenaient sénescentes quand elles étaient traitées par des principes actifs génotoxiques. Prises dans leur ensemble, ces études fournissent les données suffisantes pour conclure que les deux types de sénescence, replicative (RS) et induite par le stress (SIPS), constituent des barrières que les cellules doivent franchir pour progresser vers la malignité. À condition de garder à l'esprit que la présence prolongée de cellules sénescentes au sein d'un tissu donné pourrait promouvoir la transformation de cellules prénéoplasiques voisines (voir plus loin), ces résultats laissent évidemment entrevoir la possibilité de manipuler le processus de la sénescence à des fins thérapeutiques. 


\section{La suppression tumorale médiée par la sénescence vue de l'intérieur ou de l'extérieur}

Comme nous venons de l'indiquer, on observe des changements drastiques du profil transcriptionnel des cellules sénescentes si on les compare à des cellules quiescentes ou en phase de prolifération. Parallèlement aux stimulations et répressions respectives d'effecteurs inhibant ou stimulant la prolifération, les cellules sénescentes surexpriment une classe de gènes codant pour des protéines sécrétées comme des facteurs de croissance auto- ou paracrines, des protéines de liaison aux facteurs de croissance, des chimiokines ou des métalloprotéinases. Ce phénotype, le SASP (senescence-associated secretory phenotype), était initialement utilisé comme un simple biomarqueur du processus de sénescence, mais des découvertes récentes ont pu montrer son implication dans bien des aspects de la sénescence. De ces études, il apparaît, en outre, que la réponse sénescente dépend de réseaux protéiques à la fois intra- et extracellulaires dont la nature sera développée plus loin.

\section{Vue de l'intérieur : réactivation de p53 \\ dans les tumeurs et induction d'une sénescence massive}

Deux articles démontrent que les cancers de la souris peuvent être éliminés simplement par réactivation de la p53 [28, 29]. En utilisant des modèles animaux de cancers murins chez lesquels la fonction de p53 pouvait être manipulée de façon conditionnelle, les auteurs ont analysé son rôle dans la survie de tumeurs existantes [47]. Les auteurs observèrent une surprenante régression de la plupart des tumeurs déficientes pour p53 quasiment instantanément après la réactivation de ce gène. Selon le type de cancer, différents mécanismes entrent en jeu pour éliminer la tumeur. Les lymphomes répondent avant tout à la réexpression de p53 en déclenchant un processus apoptotique alors que les sarcomes réagissent par l'instauration d'une sénescence massive. Or, une observation remarquable est que cette régression des sarcomes associée à la sénescence fait intervenir une réaction inflammatoire produite par des polynucléaires neutrophiles, des macrophages et des cellules NK (natural killer) infiltrant la tumeur. L'analyse transcriptomique des cellules sénescentes extraites de la masse tumorale peu de temps après la réactivation de la p53 montre une stimulation des gènes codant des chimiokines pro-inflammatoires et des immunomodulateurs connus pour attirer de multiples constituants de la réponse immune innée. Étant donné la prévalence de l'inactivation de la p53 dans les cancers humains, les résultats de ces études soulignent tout l'intérêt qu'il y a à développer des stratégies thérapeutiques restaurant la fonction de la p53, et donc promotrices de la sénescence. Cependant, bien qu'impressionnants, les résultats de ces études doivent être nuancés. En effet, les tumeurs dans ces modèles se développent sur un fond $p 53$ nul pur, ce qui constitue une circonstance tout à fait artificielle. En effet, toute pression de sélection visant à muter les partenaires de l'axe p53 était totalement absente et par conséquent le développement des tumeurs était seulement dépendant de la perte de $\mathrm{p} 53$. Les analyses épidémiologiques nous apprennent toutefois que p53 est intacte dans beaucoup de tumeurs humaines alors que ces effecteurs, impliqués en amont ou en aval, sont eux-mêmes altérés. Dans de telles circonstances, la réactivation de $\mathrm{p} 53$ frapperait très probablement à côté de la cible. Enfin, et plus simplement, il n'est pas encore établi que les tumeurs humaines se comportent comme les tumeurs murines. D'autres recherches sont très certainement nécessaires pour confirmer les grandes promesses que laissent espérer les études mentionnées ci-dessus.

\section{Vue de l'extérieur: implication de protéines inflammatoires sécrétées dans le processus de sénescence}

Le recrutement de l'immunité innée n'est pas la seule modalité d'action du SASP dans la suppression tumorale. II a été mis en évidence que le SASP est nécessaire et suffisant pour établir et maintenir l'état sénescent. Une série d'études a montré que la sénescence induite soit par un oncogène activé (BRAF600) soit par le dysfonctionnement télomérique, nécessite les activités de l'IGFBP7 (insulin-like growth factor binding protein 7) ou de l'inhibiteur de l'activateur du plasminogène de type urokinase (uPAll) $[30,31,48](\rightarrow)$. Dans les deux cas, les facteurs sécrétés coopèrent directement avec le processus de sénescence, que celle-ci soit induite par un oncogène ou par un dysfonctionnement télomérique, grâce à un rétrocontrôle négatif de nature $(\rightarrow)$ Voir l'article de Marie-France GaumontLeclerc et Gerardo Ferbeyre, page 138 de ce numéro auto/paracrine atténuant l'activation des cascades MAP-kinase (IGFBP7) et PI3-kinase (UPAII). Dépourvues de ces signaux de survie, les cellules ne peuvent proliférer mais au contraire entrent en sénescence. Dans une autre série d'articles, I'IL(interleukine)-6, la chimiokine IL-8 [32] et le récepteur CXCR2 [33] sont impliqués dans un rétrocontrôle positif visant à renforcer l'arrêt en sénescence par stimulation de l'activité du facteur de transcription C/EBP $\beta$ (CCAAT/enhancer binding protein $\beta$ ). Certains auteurs ont montré l'existence d'un réseau sécrétoire auto-amplificateur renforçant la sénescence et dont les perturbations réduisent l'efficacité de cette dernière. Enfin, il a été démontré que les cellules en sénescence répondent à l'arrêt induit par les chimiokines, tandis que dans les mêmes circonstances, les cellules normales restent insensibles à de tels signaux. Cela suggère qu'un groupe autonome de chimiokines, agissant de manière autocrine, participerait à l'établissement de la sénescence. Au total, les résultats décrits ci-dessus constituent une véritable surprise car le dogme scientifique prévalant jusqu'alors soutenait que la sénescence était un processus conduit de l'intérieur des cellules et non de l'extérieur 


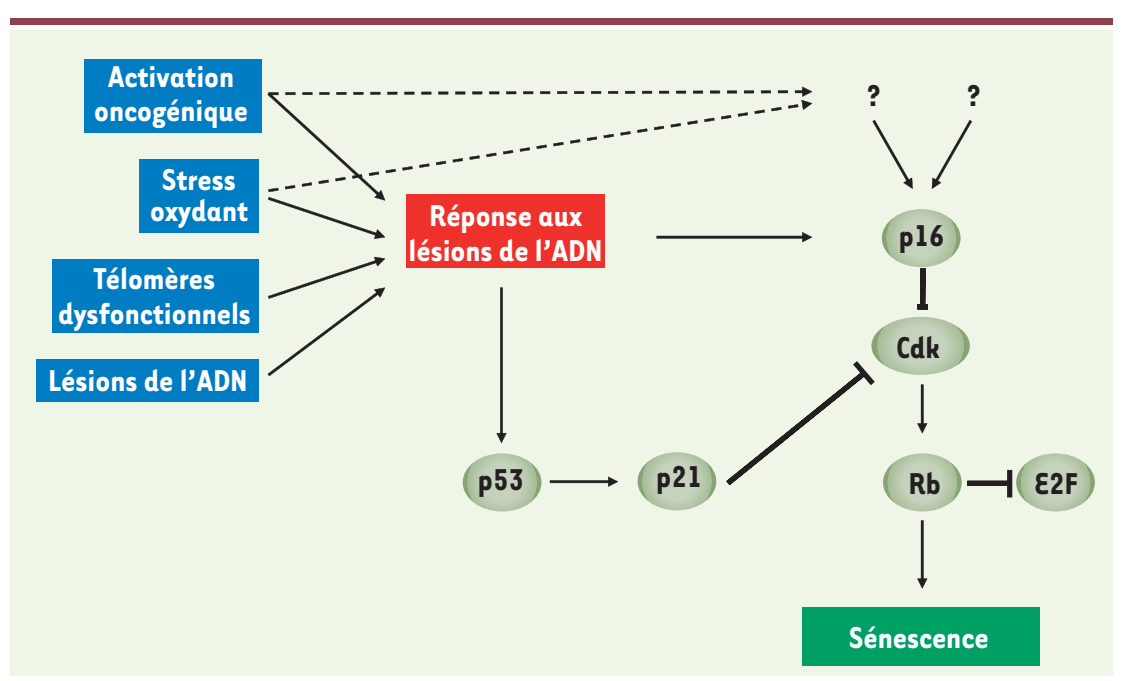

Figure 2. Voies d'induction de la sénescence dans les cellules primaires humaines. Les signaux de sénescence peuvent provoquer une réponse aux lésions de l'ADN (DDR, DNA damage response) qui se traduit par l'activation de la p53 et de sa cible p21. Ces signaux stimulent dans certains cas aussi l'expression de pl6 comme conséquence de la DDR ou par l'intermédiaire de mécanismes indépendants et encore mal connus (lignes pointillées). Les voies de signalisation de p16 et p21 convergent alors sur Rb grâce à l'inhibition des kinases dépendantes du cycle (CDK) dont le rôle est d'inactiver $\mathrm{Rb}$. L'activation de $\mathrm{Rb}$ en réponse à l'inhibition des CDK stimule fortement la sénescence en supprimant l'activité

transcriptionnelle mitogénique de E2F. Bien que les contributions respectives des voies p21 et pl6 soient mal définies, on considère que dans la plupart des cas, p16 joue un rôle crucial. Cette hypothèse est avant tout étayée par la fréquente inactivation de cette protéine durant la progression tumorale. Mais, par ailleurs, les données s'accumulent indiquant qu'une pression de sélection majeure s'exerce pour abroger ce point de contrôle de la sénescence.

(autocrine/paracrine). Par ailleurs, les différences de mode d'action des facteurs sécrétoires, évidentes à l'analyse des publications, suggèrent que les voies de signalisation déclenchant la sénescence sont très dépendantes du contexte. Par conséquent, l'universalité de ces mécanismes et leur articulation au sein des voies de sénescence déjà établies restent à évaluer.

\section{Aspect paradoxal de la sénescence, la promotion tumorale}

Une nouvelle vision des choses beaucoup moins positive entame dorénavant la réputation flatteuse de la sénescence (Figure 3). En effet, le phénotype sécrétoire des cellules sénescentes ne serait pas seulement bénéfique mais pourrait également comporter un danger pour l'organisme. Les preuves s'accumulent selon lesquelles les cellules sénescentes résiduelles au sein d'un compartiment stromal fibroblastique pourraient instaurer, via certains signaux, un environnement protumorigène pour des cellules épithéliales. De nouvelles données ont attiré l'attention sur les chimiokines et cytokines proinflammatoires comme I'IL-6, I'IL-8 et le TGF- $\beta$ (transforming growth factor $\beta$ ), et sur des métalloprotéinases, toutes à l'œuvre dans les cellules sénescentes. Elles pourraient agir de façon pro-mitogène/tumorigène et donc jouer un rôle important dans la tumorigenèse et le processus métastatique. Il est d'ailleurs remarquable que le profil d'expression de ces facteurs sécrétoires soit semblable dans les cellules sénescentes et les fibroblastes associés au cancer. Or, on sait que les fibroblastes présents dans l'environnement immédiat de la tumeur, et isolés à partir de tumeurs primaires, peuvent stimuler la croissance de cellules prénéoplasiques et favoriser la tumorigenèse [34]. De fait, des fibroblastes stromaux de tissus mammaires, quand ils sont sénescents, et non pas pré-sénescents, participent à la transformation en cellules franchement tumorigènes de cellules mammaires immortalisées mais non-néoplasiques dans un modèle murin xénogreffé. Ils sont également capables de participer à la transformation de diverses lignées néoplasiques [35]. Des études semblables, réalisées dans des modèles variés de xénogreffes, ont confirmé, depuis, ces découvertes initiales tout en limitant leur pertinence à certains organes ou tissus $[36,37]$.

\section{Une nouvelle «pléiotropie antagoniste »}

Les considérations précédentes nous laissent en apparence face à un paradoxe. D’une part, la sénescence constitue indiscutablement un mécanisme intrinsèque s'opposant à la prolifération de cellules sur le point de se transformer, d'autre part les données abondent suggérant qu'à long terme, la présence résiduelle de cellules sénescentes pourrait altérer le microenvironnement et promouvoir la tumorigenèse dans un épithelium prénéoplasique lui-même non sénescent. Ce type de paradoxe n'est pas sans précédent dans les théories de l'évolution où il apparaît sous le nom de «pléiotropie antagoniste ». Selon cette théorie, la sénescence serait interprétable comme un mécanisme préservant les individus des tumeurs tant que ceux-ci sont dans leur période d'activité reproductrice durant laquelle s'exerce la pression de sélection. Ce processus pourrait par la suite avoir des conséquences néfastes lorsque les pressions de sélection ne s'exercent plus (ombre évolutive), c'est-à-dire dans la phase post-reproductive de la vie, période où les cellules sénescentes potentiellement délétères ont fini par s'accumuler 


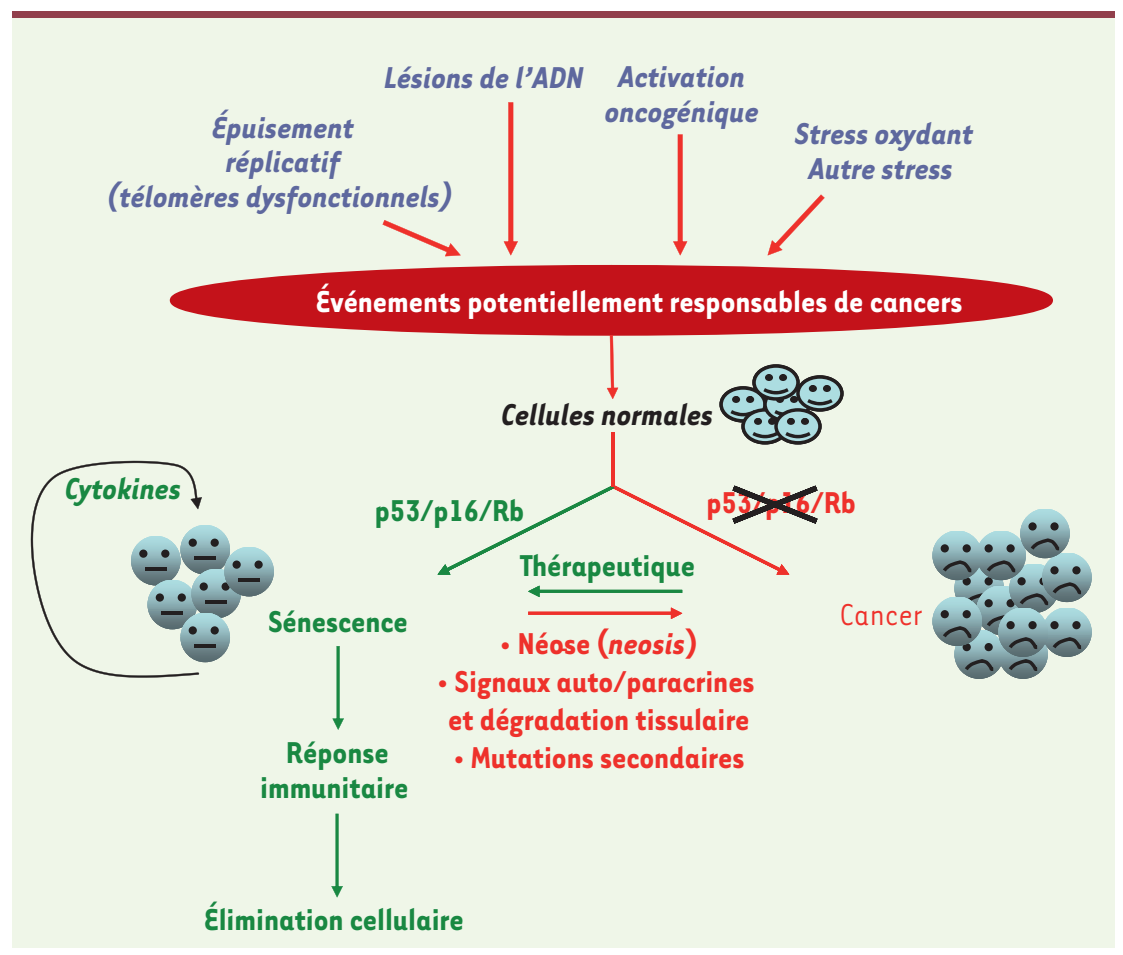

Figure 3. Une vision synthétique de la sénescence dans la suppression et la promotion tumorales. Des signaux transmis par un disfonctionnement télomérique, des lésions de I'ADN, l'activation d'oncogènes ou d'autres stress peuvent provoquer la sénescence (ou l'apoptose non incluse ici) dans des cellules normales après mobilisation des suppresseurs de tumeur p53 ou p16/Rb. La sénescence est renforcée par un rétrocontrole positif médié par des cytokines. De plus, elle peut stimuler l'immunité innée et l'élimination des cellules abimées. L'inactivation de $\mathrm{p} 53$ et de $\mathrm{pl6} / \mathrm{Rb}$ qui se produit souvent durant la carcinogénèse abroge la sénescence et établit un milieu favorable à la transformation. Toutefois, même les cellules cancéreuses peuvent adopter, de façon stable ou non, un état analogue à la sénescence sous l'action de composés génotoxiques. Les cellules cancéreuses sénescentes peuvent potentiellement retourner vers l'état prolifératif grâce à la néose, l'inactiva-

tion de facteurs oncosuppresseurs ou l'activation de certains oncogènes. Enfin, les cellules sénescentes peuvent elles-aussi établir un environnement favorable à la tumeur grâce à la dégradation tissulaire et production de signaux auto/paracrines (voir texte pour les détails).

dans l'organisme [38] (Figure 3). De fait, plusieurs études ont montré que les cellules sénescentes s'accumulent effectivement dans les compartiments stromaux des individus âgés où elles altèrent profondément les caractéristiques de croissance des cellules épithéliales prénéoplasiques [39]. La question est maintenant de savoir comment séparer les «bons » et les «mauvais » aspects de la sénescence dans une tumeur donnée. De plus, nous devons déterminer si l'arrêt en sénescence d'une cellule tumorale traitée par un principe actif cytotoxique est de la même qualité que celui des cellules en RS ou en SIPS.

\section{L'état sénescent est-il définitif?}

Nous avons vu que la sénescence est une puissante barrière bloquant le développement de cellules tumorales non décelables. La question se pose de savoir comment ces cellules tumorales court-circuitent la sénescence dans certaines circonstances et progressent ensuite vers la malignité la plus aboutie. En raison de l'espace restreint dévolu à cette revue, nous limiterons la discussion aux mécanismes d'inversion de la sénescence.

\section{Diversité des processus de maintien de la sénescence}

On croyait initialement que la sénescence était un mécanisme irréversible d'arrêt du cycle cellulaire. Ce dogme a été remis en question au cours des cinq dernières années à la suite de la découverte de différences qualitatives dans les divers types d'arrêt en sénescence. Un travail remarquable de Judith Campisi et de ses collaborateurs a permis d'établir en 2003 que différents types de fibroblastes humains pri- maires varient dans leurs mécanismes d'établissement et de maintien de la sénescence [40]. Dans certaines cellules, le mécanisme qui prédomine pour l'initiation et le maintien de la sénescence en réponse à un stress est strictement dépendant de p53 alors que dans d'autres cellules, p53 et l'axe Rb-pl6 sont tous deux impliqués dans le déclenchement et la stabilisation de l'état sénescent. Ces découvertes renforcent un peu plus l'hypothèse selon laquelle une sénescence purement p53dépendante peut être inversée par l'inactivation de p53 alors que, une fois $\mathrm{Rb}$ et $\mathrm{pl} 6$ complètement mobilisés, la sénescence devient quasiment irréversible, et ne peut pas être abrogée par l'inactivation de Rb ou pl6. II faut noter que dans les cellules sénescentes où l'axe Rb-pl6 prédomine, l'inactivation de $\mathrm{Rb}$ réinitie la synthèse d'ADN mais échoue à relancer la prolifération, indiquant que $\mathrm{Rb}$ avait imposé au préalable un deuxième verrou sur le point de contrôle G2-M du cycle cellulaire. Chez la souris, il a été montré que l'inactivation simultanée des trois membres de la famille Rb (p107, pl10 et p130) dans les cellules sénescentes est capable de relancer la prolifération ce qui souligne des différences fondamentales entre homme et souris au regard de la stricte dépendance vis-à-vis de $\mathrm{Rb}$ du processus de sénescence [41]. De fait, il a été démontré que l'axe Rb-pl6 est impliqué dans la formation d'un blocage de la cytokinèse par une réduction de l'activité de la kinase de 
sortie du cycle WARTS/LATS1 (large tumor suppressor 1) [42]. Cette situation montre clairement que différents types de cellules humaines peuvent varier dans leur propension à stimuler pl6 et par conséquent, activer $\mathrm{Rb}$. Quoi qu'il en soit, les données disponibles indiquent que pl6 est cruciale pour assurer l'irréversibilité de la sénescence ce qui renforce encore son importance en tant qu'oncosuppresseur (Figure 3).

\section{Le piège de la néose}

Nous avons déjà mentionné l'inductibilité du phénotype sénescent dans les cellules cancéreuses exposées à un principe actif génotoxique. II devient de plus en plus évident que considérer l'induction de la sénescence comme une thérapeutique anticancéreuse recèle certains pièges. L'un d'entre eux, déjà décrit plus haut, est lié à l'instauration possible d'un environnement protumorigène par les cellules sénescentes quand celles-ci ne sont pas complètement éliminées. L'autre inconvénient est dû au fait que les cellules tumorales engagées dans la sénescence sous l'effet d'un principe actif, et parfois même les cellules normales sénescentes, semblent capables à la fin de leur vie d'échapper à la sénescence par un mécanisme appelé la néose [43, 44]. Jusqu'à ce jour la néose a été largement ignorée en tant que mécanisme permettant le retour des cellules sénescentes vers l'état tumoral. Ce mécanisme doit d'ailleurs démontrer sa validité dans les tumeurs primaires. Néanmoins, le concept de néose mérite, à notre avis, que l'on s'y intéresse de façon plus approfondie à l'avenir. Pour cette raison nous en ferons une brève description.

La néose est une forme particulière de division cellulaire, bien différente de la mitose, qui se produit uniquement dans des cellules sénescentes endopolyploïdes. La néose est une division de type parasexuelle avec une réduction chromatique analogue à la méiose. On l'observe dans certaines cellules géantes multinucléées et/ou polyploïdes. Les cellules mères de la néose (NMC) sont dotées de génomes non-viables et polyploïdes. Elles génèrent des quantités variables de petites cellules mononucléées actives sur le plan mitotique, appelées cellules Raju, par un mécanisme de bourgeonnement nucléaire suivi d'une cytokinèse asymétrique. Comme les cellules Raju sont produites par bourgeonnement nucléaire, les contrôles du fuseau mitotique sont absents, conduisant à une aneuploïdie elle-même génératrice d'une forte instabilité génomique dans les cellules filles des cellules Raju. Le cycle cellulaire des cellules Raju ressemble à celui des cellules embryonnaires précoces et elles peuvent transitoirement exprimer des marqueurs de cellules souches. Leur descendance par mitose peut connaître plusieurs cycles de néose, ce qui aboutit à un rajeunissement périodique et à une forte hétérogénéité de la population de cellules tumorales.

\section{Conclusion}

La dernière décennie a vu l'accomplissement de grands progrès dans la compréhension des mécanismes génétiques de régulation de la sénescence cellulaire et de son fonctionnement en tant que puissant mécanisme de suppression des tumeurs. Cette recherche ouvre potentiellement de nouvelles approches thérapeutiques, mais doit encore faire l'objet d'une exploration rigoureuse au cours des prochaines années. Les thérapeutiques d'induction de la sénescence notamment, doivent faire l'objet d'un examen scrupuleux car les cellules sénescentes seraient dans certains cas dotées d'une activité protumorigène, et, à l'heure actuelle, il n'est toujours pas possible de déterminer parmi ces cellules lesquelles seront favorables à la suppression tumorale et lesquelles ne le seront pas. Les années qui viennent apporteront, à n'en pas douter, des informations décisives quant au rôle véritablement protecteur ou plutôt délétère des cellules sénescentes vis-à-vis de la tumorigenèse. $\diamond$

\section{SUMMARY}

A re-view of cellular senescence:

friend or foe of tumorigenesis?

Cellular senescence, like apoptosis, is now widely accepted as a potent tumor suppressor mechanism operating in normal mitotic mammalian cells. Originally, it was identified as a process that limits the replicative lifespan of primary human cells in culture because of telomere attrition and was therefore termed "replicative" senescence (RS). However, previous findings have demonstrated that a phenotype indistinguishable from replicative senescence, collectively called "stress-induced premature" senescence (SIPS), can be induced without extensive cell division in normal as well as cancer cells by a variety of stresses and signaling imbalances. Recent developments have also indicated that, despite their tumor-suppressive capacity, senescent cells themselves could produce neoplastic cells under certain circumstances and promote the growth of preneoplastic cells, raising the possibility that senescence might function as a biological "Trojan" horse. Here, we take a snapshot of the progress in understanding the causes and consequences of cellular senescence in vitro and in vivo. $\diamond$

\section{RÉFÉRENCES}

1. Campisi J, D’Adda di Fagagna F. Cellular senescence: when bad things happen to good cells. Nat Rev Mol Cell Biol $2007 ; 8: 729-40$.

2. Deng $Y$, Chan SS, Chang S. Telomere dysfunction and tumour suppression: the senescence connection. Nat Rev Cancer 2008; $8: 450-8$.

3. Prieur A, Peeper DS. Cellular senescence in vivo: a barrier to tumorigenesis. Curr Opin Cell Biol $2008 ; 20: 150-5$.

4. Braig M, Schmitt CA. Oncogene-induced senescence: putting the brakes on tumor development. Cancer Res $2006 ; 66: 2881-4$.

5. Shay JW, Roninson IB. Hallmarks of senescence in carcinogenesis and cancer therapy. Oncogene $2004 ; 23: 2919-33$.

6. Schmitt CA. Cellular senescence and cancer treatment. Biochim Biophys Acta 2007 ; $1775: 5-20$. 


\section{GLOSSAIRE}

C/EBP $\boldsymbol{\beta}$ : CCAAT/ enhancer binding protein $\beta$

HMG : high mobility group

HP1 : heterochromatin protein 1

IGFBP : insulin-like growth factor binding protein

NK : natural killer

NMC : cellules mères de la néose

PCNA : proliferation cell nuclear antigen

PIASy : protein inhibitor of activated STAT

PML : promyelocytic leukemia

RS : sénescence réplicative

SA- $\beta$-Gal : $\beta$-galactosidase associée à la sénescence

SAHF : foyers hétérochromatiniens associés à la sénescence

SASP : senescence-associated secretory phenotype, phénotype sécrétoire associé à la sénescence

SDF : senescence-associated DNA damage foci, foyers de lésion de I'ADN associés à la sénescence

SIPS : sénescence prématurée induite par le stress

TGF- $\boldsymbol{\beta}$ : transforming growth factor $\boldsymbol{\beta}$

TIF : telomere dysfunction-induced foci, foyers lésionnels induits par les télomères dysfonctionnels

7. Collado M, Serrano M. The senescent side of tumor suppression. Cell Cycle $2005 ; 4: 1722-4$.

8. Hanahan D, Weinberg RA. The hallmarks of cancer. Cell $2000 ; 100: 57-70$.

9. Adams PD. Remodeling chromatin for senescence. Aging Cell $2007 ; 6: 425-62$.

10. Narita M. Cellular senescence and chromatin organisation. Br J Cancer $2007 ; 96: 686-91$.

11. Bischof 0, Dejean A. SUMO is growing senescent. Cell Cycle $2007 ; 6: 677-81$.

12. Sedivy JM. Telomeres limit cancer growth by inducing senescence: long-sought in vivo evidence obtained. Cancer Cell $2007 ; 11: 389-91$.

13. Di Micco R, Fumagalli $M$, Cicalese $A$, et al. Oncogene-induced senescence is a DNA damage response triggered by DNA hyper-replication. Nature $2006 ; 444: 638-42$.

14. Bartkova J, Rezaei N, Liontos M, et al. Oncogene-induced senescence is part of the tumorigenesis barrier imposed by DNA damage checkpoints. Nature 2006 ; $444: 633-7$.

15. Takai H, Smogorzewska A, de Lange T. DNA damage foci at dysfunctional telomeres. Curr Biol $2003 ; 13: 1549-56$

16. Herbig U, Jobling WA, Chen BP, et al. Telomere shortening triggers senescence of human cells through a pathway involving ATM, p53, and p21(CIP1), but not p16(INK4a). Mol Cell 2004 ; $14: 501-13$.

17. Reaper PM, di Fagagna F, Jackson SP. Activation of the DNA damage response by telomere attrition: a passage to cellular senescence. Cell Cycle $2004 ; 3: 543-6$.

18. Smogorzewska A, de Lange T. Different telomere damage signaling pathways in human and mouse cells. EMBO J $2002 ; 21: 4338-48$.

19. Young AP, Schlisio S, Minamishima YA, et al. VHL loss actuates a HIF-independent senescence programme mediated by $\mathrm{Rb}$ and $\mathrm{p} 400$. Nat Cell Biol $2008 ; 10: 361-9$.

20. Collado M, Gil J, Efeyan A, et al. Tumour biology: senescence in premalignant tumours. Nature $2005 ; 436: 642$.

21. Chen Z, Trotman LC, Shaffer D, et al. Crucial role of $\mathrm{p} 53$-dependent cellular senescence in suppression of Pten-deficient tumorigenesis. Nature $2005 ; 436$ : 725-30.

22. Michaloglou C, Vredeveld LC, Soengas MS, et al. BRAFE600-associated senescence-like cell cycle arrest of human naevi. Nature $2005 ; 436: 720-4$.

23. Braig M, Lee $\mathrm{S}$, Loddenkemper $\mathrm{C}$, et al. Oncogene-induced senescence as an initial barrier in lymphoma development. Nature $2005 ; 436: 660-5$.

24. Feldser DM, Greider CW. Short telomeres limit tumor progression in vivo by inducing senescence. Cancer Cell $2007 ; 11: 461-9$.

25. Guo X, Deng $Y$, Lin $Y$, et al. Dysfunctional telomeres activate an ATM-ATR-dependent DNA damage response to suppress tumorigenesis. EMBO J $2007 ; 26: 4709-19$.

26. Londono-Vallejo $\mathrm{A}$, Lenain $\mathrm{C}$, Gilson $\varepsilon$. Cibler les télomères pour forcer les cellules cancéreuses à rentrer en sénescence. Med Sci (Paris) 2008 ; 24 : 383-9.
27. Te Poele RH, Okorokov AL, Jardine L, et al. DNA damage is able to induce senescence in tumor cells in vitro and in vivo. Cancer Res $2002 ; 62$ : 1876-83.

28. Xue W, Zender L, Miething C, et al. Senescence and tumour clearance is triggered by p 53 restoration in murine liver carcinomas. Nature 2007; $445: 656-60$.

29. Ventura A, Kirsch DG, McLaughlin $M E$, et al. Restoration of $p 53$ function leads to tumour regression in vivo. Nature $2007 ; 445: 61-5$.

30. Wajapeyee N, Serra RW, Zhu X, et al. Oncogenic BRAF induces senescence and apoptosis through pathways mediated by the secreted protein IGFBP7. Cell 2008; $132: 363-74$.

31. Kortlever RM, Higgins PJ, Bernards R. Plasminogen activator inhibitor-1 is a critical downstream target of $\mathrm{p} 53$ in the induction of replicative senescence. Nat Cell Biol $2006 ; 8: 877-84$.

32. Kuilman T, Michaloglou C, Vredeveld LC, et al. Oncogene-induced senescence relayed by an interleukin-dependent inflammatory network. Cell 2008; 133: 1019-31.

33. Acosta JC, O'Loghlen A, Banito A, et al. Chemokine signaling via the CXCR2 receptor reinforces senescence. Cell $2008 ; 133$ : 1006-18.

34. Olumi AF, Grossfeld GD, Hayward SW, et al. Carcinoma-associated fibroblasts direct tumor progression of initiated human prostatic epithelium. Cancer Res 1999; 59 : 5002-11.

35. Krtolica A, Campisi J. Integrating epithelial cancer, aging stroma and cellular senescence. Adv Gerontol $2003 ; 11: 109-16$.

36. Liu D, Hornsby PJ. Fibroblast stimulation of blood vessel development and cancer cell invasion in a subrenal capsule xenograft model: stressinduced premature senescence does not increase effect. Neoplasia 2007; $9: 418-26$.

37. Liu D, Hornsby PJ. Senescent human fibroblasts increase the early growth of xenograft tumors via matrix metalloproteinase secretion. Cancer Res $2007 ; 67: 3117-26$.

38. Campisi J. Senescent cells, tumor suppression, and organismal aging: good citizens, bad neighbors. Cell $2005 ; 120: 513-22$.

39. Dean JP, Nelson PS. Profiling influences of senescent and aged fibroblasts on prostate carcinogenesis. Br J Cancer $2008 ; 98: 245-9$.

40. Beausejour CM, Krtolica A, Galimi F, et al. Reversal of human cellular senescence: roles of the $\mathrm{p} 53$ and $\mathrm{p} 16$ pathways. EMBO J 2003; $22: 4212-22$

41. Sage J, Miller AL, Perez-Mancera PA, et al. Acute mutation of retinoblastoma gene function is sufficient for cell cycle re-entry. Nature $2003 ; 424: 223-8$.

42. Takahashi A, Ohtani N, Yamakoshi K, et al. Mitogenic signalling and the pl6INK4a-Rb pathway cooperate to enforce irreversible cellular senescence. Nat Cell Biol 2006; 8 : 1291-7.

43. Rajaraman R, Guernsey DL, Rajaraman MM, Rajaraman SR. Stem cells, senescence, neosis and self-renewal in cancer. Cancer Cell Int 2006 ; $6: 25$.

44. Erenpreisa J, Cragg MS. Cancer: a matter of life cycle? Cell Biol Int 2007 ; $31: 1507-10$.

45. Gire V. La sénescence : une barrière télomérique à l'immortalité ou une réponse cellulaire aux stress physiologiques? Med Sci (Paris) 2005; $21: 491-7$

46. Chien WW, Ffrench M. Régulation de p161NK4a, sénescence et oncogenèse. Med Sci (Paris) $2006 ; 22: 865-71$.

47. Toledo F, Bluteau 0 , Simeonova I. Réactivation de $p 53$ dans les tumeurs: une stratégie antitumorale prometteuse. Med Sci (Paris) 2007 ; $23: 565-7$.

48. Gaumont-Leclerc MF, Ferbeyre G. Les cytokines préviennent les tumeurs via un mécanisme de sénescence cellulaire. Med Sci (Paris) 2009; $25: 138-40$.

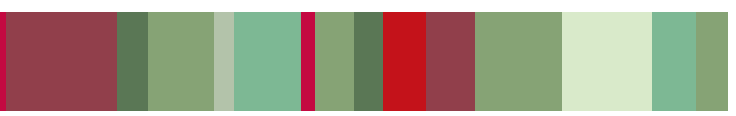

TIRÉS À PART

O. Bischof 